\title{
Application of image analysis for the measurement of liquid metal free surface
}

\author{
S. Golak \\ Faculty of Materials Science and Metallurgy, \\ Department of Electrotechnology, Silesian University of Technology, \\ Poland
}

\begin{abstract}
During the induction melting and stirring of molten metal a meniscus forms on the surface of the bath. This phenomenon affects the processes occurring on the metal-gas interface since they change the size of the real free surface of the metal. Numerical simulations indicate a significant scale of the phenomenon during typical processes of the induction melting of metals. Experimental measurements of the real free surface of induction melted metals can be quite difficult because of the rapid changes in the surface shape and high temperature of the molten metal. Therefore, a fast non-contact method with the use of laser and image analysis was proposed. However, in the case of liquid metals an optical method of measurement has its limitations as many molten metals shine. The problem was resolved by the use of a green laser and a narrow-band optical filter. This paper presents the methodology and problems of meniscus shape non-contact measurements.
\end{abstract}

Keywords: liquid metal, free surface, laser measurement.

\section{Introduction}

The processes of induction melting, stirring and refining of liquid metals in crucibles and ladles are becoming more and more popular in the metallurgical industry. This situation promotes constant development of the equipment used in the above-mentioned processes, which in turn means continuous improvement of the designing methods. One of these is to expand the potential of simulating metallurgical processes and indeed it can be observed nowadays that more and more physical phenomena are taken into account in such simulations. At the 
beginning just one-dimensional or quasi two-dimensional analytical models of electromagnetic and temperature fields were constructed. Then, together with the development of computer science, there appeared numerical tools for simulation of these fields and their coupling. Greater availability of computers and higher computing power contributed to progress in computational fluid dynamics, which made it possible to simulate liquid metal hydrodynamics occurring in real metallurgical devices. Initially, the simulations of electromagnetic and hydrodynamic fields were conducted as separate stages. First, the distribution of electromagnetic forces acting on the metal was calculated, and it was followed by the determination of the liquid metal velocity field induced by these forces. Those simulations assumed that the geometry of the liquid metal is not subject to change. The most frequent assumption was even that the surface of the liquid metal was perfectly flat.

In fact, each metal forms on its surface a meniscus being the resultant of the surface energy of the crucible walls and total surface tension of the liquid metal. This process intensifies significantly under the influence of an electromagnetic field. The geometry of the liquid metal is distorted, and in effect the actual contact area between the metal and the atmosphere is bigger than in the case of a flat surface (Blacha et al. [1], Golak and Przylucki [2,3]). The simulations conducted in our department have proved that the surface area after deformation might even be increased by as much as 150 percent.

Information of the real shape that the surface assumes is of great significance in the analysis of the melting, stirring and refining processes. During metal melting and heating it is the geometry of the melt that determines the actual power emitted in the charge, and consequently the capacity of the equipment in question. The distribution of the velocity field in induction stirrers determined for a flat free surface will be substantially different from the distribution for the deformed surface. Yet, the greatest importance of a free surface can be seen in the refining and other processes occurring at the interface of the phase's liquid metal-atmosphere. Processes of this type show almost linear dependency on the actual size of the metal free surface. For this reason inaccurate estimation of the bath shape may completely distort the results of the most accurate simulations.

The above-mentioned simulations conducted in our department revealed the scale of the phenomenon and its significance. However, the results of numerical simulation should be verified experimentally in order to validate the methodology applied and make any necessary corrections. To do this the measurements of the real shape of the bath must be performed and compared with the simulation results. In this way not only the calculations of the bath shape may be verified, but also, indirectly, the calculations of the electromagnetic forces and velocity field can be confirmed as the obtained meniscus is a resultant of the processes represented by these simulations.

Measurements of a liquid metal are by no means an easy task because of its high temperature and chemical activity. That is why non-contact methods are often preferred.

The level of liquid metal may be determined by point measurement performed with the use of radar, ultrasonic, and recently also laser sensors. 
Within the range of applications discussed, however, the point measurement proves to be insufficient. It turns out to be necessary that the whole shape of the liquid metal should be scanned with adequate resolution. The paper presents an attempt to adapt a widely used technique of 3D laser scanning to measure the geometry of a liquid metal in induction crucibles.

\section{Method description}

Laser measurement of the level is most often performed by triangulation method because of its accuracy and relative simplicity (e.g. Mass et al. [4]). It consists in a projection of a laser beam at a certain angle onto the surface from which the distance is to be measured and the camera recording of the beam reflection.

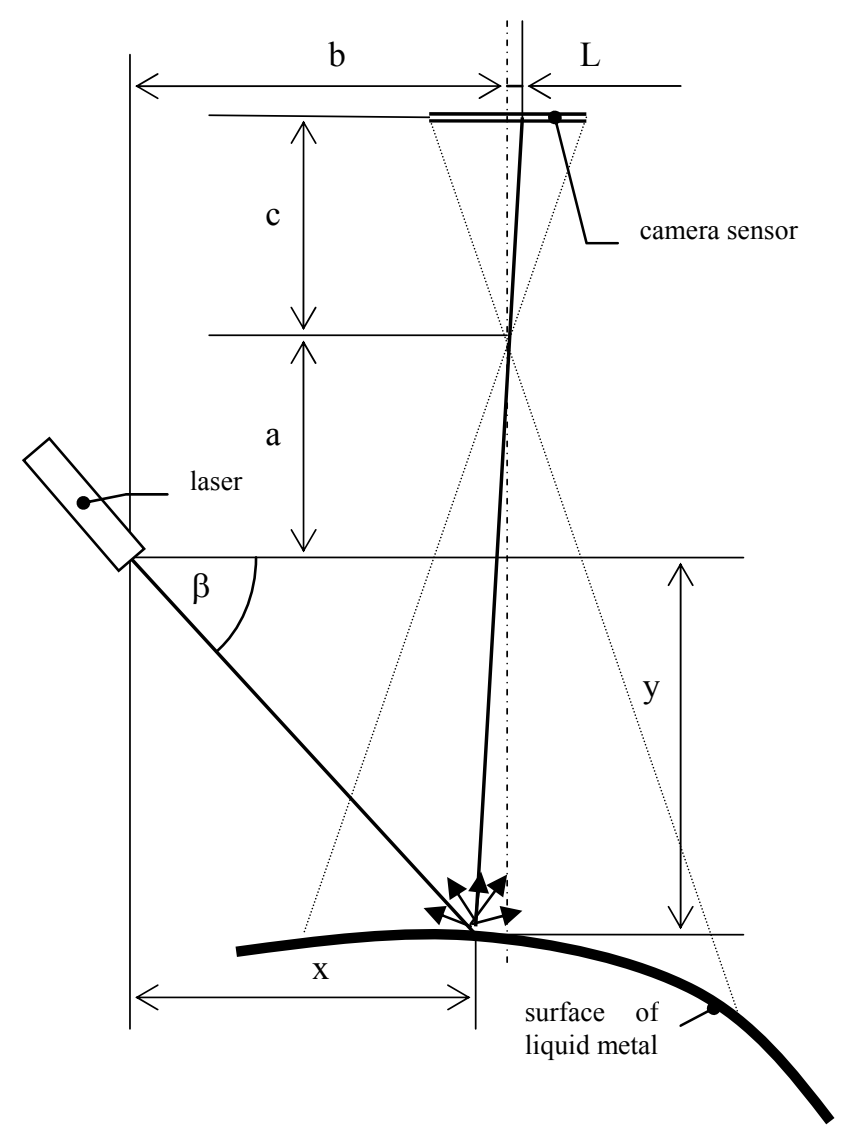

Figure 1: Level measurement by the method of diffused light recording. 
Two techniques of measurement may be distinguished here. In one of them it is the light diffused on the surface that is recorded, while in the other it is the beam reflected from the surface and projected onto the screen.

The choice between the above-mentioned methods of level measurement (and consequently surface profile measurement) of the liquid metal will depend on several factors. The first method is applicable when the laser beam is diffused on the surface of the liquid metal, which occurs when various kinds of impurities, micro-bubbles and the like can be found on it. Despite the inconvenience, the method is widely used for the simplicity of the measurement procedure.

Figure 1 presents a schematic diagram of the measurement of metal level by the method of diffused light recording. As can be seen, a shift in the level of liquid causes a shift of the recorded light spot of the laser beam. Coordinates of the surface point can be estimated by the solution of equation set (1).

$$
\left\{\begin{array}{l}
y=\frac{c(b-x)}{L}-a \\
y=\tan (\beta) x
\end{array}\right.
$$

where:

$x, y$ - coordinates of the surface point

$\beta$ - the tilt of the laser

$L$ - the position of the laser beam recorded by the camera

$a, b, c$ - geometric values (see fig. 1)

In the case of some metals which melt at high temperatures (e.g. copper) a problem of their shining occurs. The spectrum of this light has a distribution similar to the light of a black body (fig. 2) described by Planck's law.

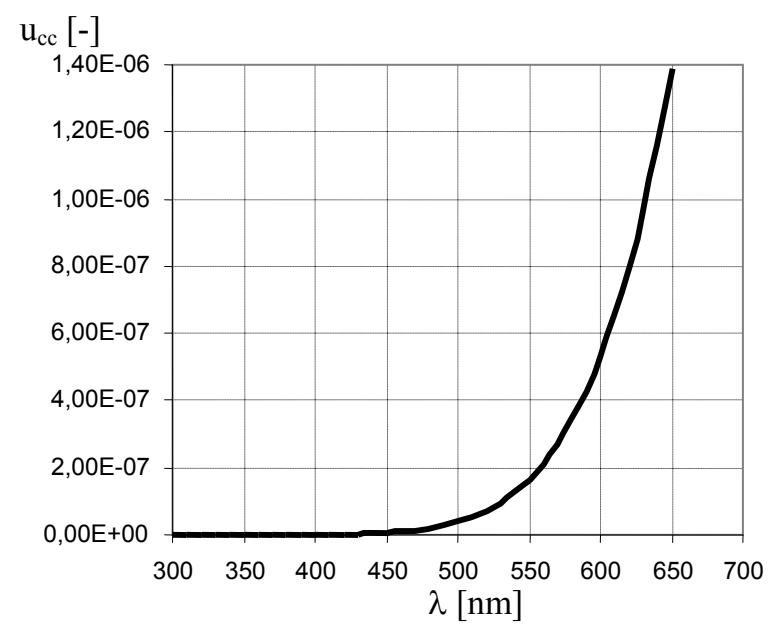

Figure 2: Normalized Planck distribution for the temperature of liquid copper. 
Laser light reflected from the metal surface is then suppressed and its recording is not possible. The solution here is a narrow-band filter on the camera lens that will remove the spectrum components radiated by the metal and leave only the part of spectrum components in which the laser beam light is contained. Semiconductor red lasers, which are most frequently used, emit light of wavelength $650 \mathrm{~nm}$, which is contained in the part of spectrum radiated by the heated metal. For this reason, a good idea is to use a laser with a shorter wavelength is suggested. Green semiconductor laser with wavelength of $532 \mathrm{~nm}$ have been recently available on the market. Within this range of radiation hot metal shines less brightly, and owing to filtration it is easier to distinguish between the laser light and metal shine.

The method of point measurement of the liquid metal can be extended to linear measurement. In order to do this a dot line must be projected onto the metal surface. In 3D laser scanners a mechanical system deflecting the laser beam is commonly used. In the case of induction devices, however, quickly changing processes have to be often recorded. Having in mind fast digital cameras so easily available today, it must be concluded that the mechanical system would become the factor limiting the speed of measurement.

Therefore what was applied in the presented solution was multiplication of laser beam by an optic method based on interference grid. The selection of a suitable head allows a dot line to be projected onto the surface of the liquid metal. Since the induction machines considered here are usually characterized by full axial symmetry, the measurement of the radial component of the curvature makes it possible to determine full geometry of the surface.

The optic method can also be used to project a dot matrix, which will allow a full scanning of the surface.

When the laser ray is not diffused on the metal surface, the method of the reflected beam should be applied. Figure 3 shows the idea of this measurement. Coordinates of the surface point are estimated by solution of equation set (2).

$$
\left\{\begin{array}{l}
y=b+H+(a-x) \tan (\beta-2 \alpha) \\
y=x \tan (\beta)
\end{array}\right.
$$

where:

$x, y$ - coordinates of the surface point

$\alpha$ - the tilt of surface

$\beta$ - the tilt of the laser

$H$ - the position of the laser beam spot on the projection panel recorded by the camera

$a, b-$ geometric values (see fig. 3 )

The technique is sensitive to the curvature of the surface from which the distance is measured. As Figure 4 presents, even small tilt of the surface which reflects the beam causes a significant change in its direction. A shift of the light spot on the screen resulting from the change of the beam direction may be far more serious than the shift resulting from the change in the metal level. 
Because in the application considered a meniscus occurs on the surface of the liquid metal, the method of point measurement based on the reflected beam is completely useless. However, multipoint measurement will allow the calculation of the metal surface curvature on the basis of the measurements performed by the method of reflected beam, according to solution of equation set (3).

$$
\begin{aligned}
& \begin{cases}y_{i}=b+H_{i}+\left(a-x_{i}\right) \tan \left(\beta_{i}-2 \alpha_{i}\right) & 0 \leq i \leq N \\
y_{i}=x_{i} \tan \left(\beta_{i}\right) & 0 \leq i \leq N \\
\tan \left(\alpha_{i}\right)=y_{i-1} \frac{2 x_{i}-x_{i}-x_{i+1}}{\left(x_{i-1}-x_{i}\right)\left(x_{i-1}-x_{i+1}\right)}+ & 0<i<N\end{cases} \\
& y_{i} \frac{2 x_{i}-x_{i-1}-x_{i+1}}{\left(x_{i}-x_{i-1}\right)\left(x_{i}-x_{i+1}\right)}+y_{i+1} \frac{2 x_{i}-x_{i-1}-x_{i}}{\left(x_{i+1}-x_{i-1}\right)\left(x_{i+1}-x_{i}\right)} \\
& \tan \left(\alpha_{0}\right)=y_{0} \frac{2 x_{0}-x_{1}-x_{2}}{\left(x_{0}-x_{1}\right)\left(x_{0}-x_{2}\right)}+y_{1} \frac{2 x_{0}-x_{0}-x_{2}}{\left(x_{1}-x_{0}\right)\left(x_{1}-x_{2}\right)}+ \\
& \left\{y_{2} \frac{2 x_{0}-x_{0}-x_{1}}{\left(x_{2}-x_{0}\right)\left(x_{2}-x_{1}\right)}\right. \\
& \tan \left(\alpha_{N}\right)=y_{N-2} \frac{2 x_{N}-x_{N-1}-x_{N}}{\left(x_{N-2}-x_{N-1}\right)\left(x_{N-2}-x_{N}\right)}+ \\
& y_{N-1} \frac{2 x_{N}-x_{N-2}-x_{N}}{\left(x_{N-1}-x_{N-2}\right)\left(x_{N-1}-x_{N}\right)}+y_{N} \frac{2 x_{N}-x_{N-2}-x_{N-1}}{\left(x_{N}-x_{N-2}\right)\left(x_{N}-x_{N-1}\right)} \\
& y_{0} \frac{-x_{1}-x_{2}}{\left(x_{0}-x_{1}\right)\left(x_{0}-x_{2}\right)}+y_{1} \frac{-x_{0}-x_{2}}{\left(x_{1}-x_{0}\right)\left(x_{1}-x_{2}\right)}+ \\
& y_{2} \frac{-x_{0}-x_{1}}{\left(x_{2}-x_{0}\right)\left(x_{2}-x_{1}\right)}=0
\end{aligned}
$$

where:

$x_{i}, y_{\mathrm{i}}$ - coordinates of the $\mathrm{i}$-th surface point

$\alpha_{i}$ - the tilt of surface estimated from derivative of parabolically approximated function of shape

$\beta_{i}$ - the tilt of the laser i-th laser beam

$H_{i}$ - the position of the i-th laser beam spot on the projection panel recorded by the camera

$a, b$ - geometric values

$N$ - a number of laser beams and estimated surface points.

The last equation of the set represents the known, zero value of the surface tilt on the axis of the crucible.

Unfortunately, although the application of the above method solves the problem of the meniscus curvature effecting the measurement of the beam 
reflection, it does not eliminate the errors resulting from local changes in the tilt of the surface. The solution to this problem lies in the assumption that the curvature of the surface caused by the meniscus is subject to changes much slower than the local deformations in the surface shape. This method relies on the measurement of the surface shape in a given period of time (short enough to record momentary shape of the meniscus), and then averaging the obtained result in time.

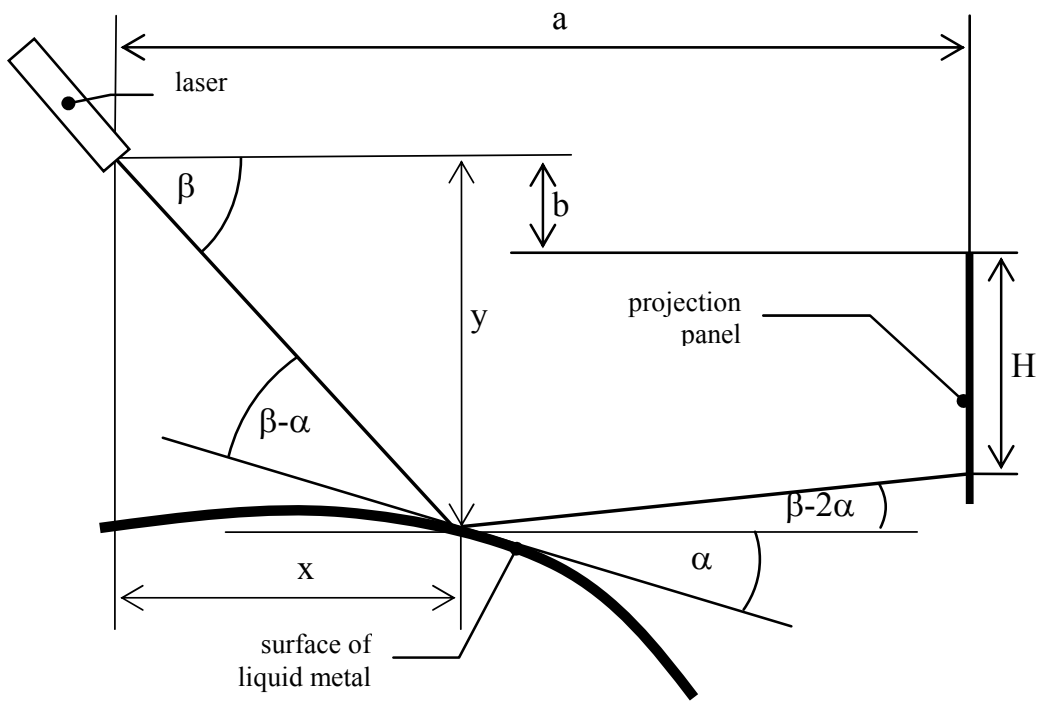

Figure 3: Level measurement by the method of reflected light recording.

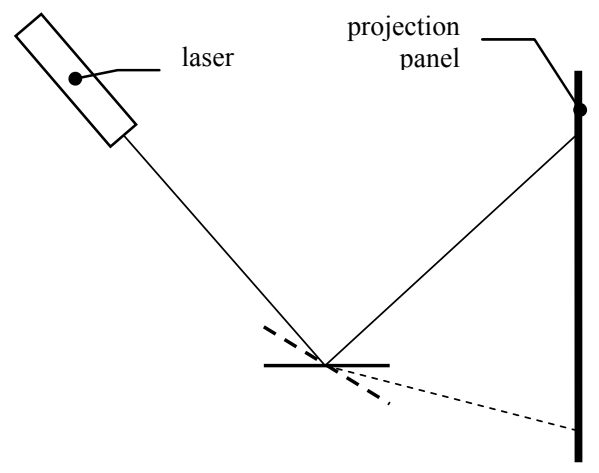

Figure 4: Influence of surface tilt. 


\section{Method application}

On the basis of the measurement methods developed an experimental setup was designed where the technique of diffused beam was applied.

A green semiconductor laser with wavelength of $532 \mathrm{~nm}$ was used. The model chosen for recording of the light (both reflected and diffused) was a colour camera, uEye UI-1640-C, resolution $1280 \times 1024$, with CMOS matrix and scanning frequency of $25 \mathrm{~Hz}$ with full resolution and the possibility of increasing the frequency (at the expense of resolution) do $254 \mathrm{~Hz}$. The camera was equipped with the lens Pentax C1614-M with focal length of $16 \mathrm{~mm}$. So instrumented, the camera will monitor an area of $12 \mathrm{~cm}$ by $10 \mathrm{~cm}$ from the distance of $0,5 \mathrm{~m}$. A $532 \mathrm{~nm}$ narrow-band interference filter with bandwidth of $3 \mathrm{~nm}$ was also added to the measurement system.

Figure 5 presents the arrangement of the stand for the measurement by the method of diffused beam. Theoretical accuracy of the measurement of coordinates in this arrangement can be calculated from eqn (4) and is equal to about $10^{-4} \mathrm{~m}$. In reality, the accuracy is reduced along with the increase in the tilt of the metal surface because of the blurring of the light spot. For this reason the lowest accuracy is obtained nearby the crucible walls.

$$
|\Delta y|=\left|\frac{-a c \cdot \operatorname{ctg}(\beta)-b c}{(L+c \cdot \operatorname{ctg}(\beta))^{2}} \cdot\right| \Delta L|| ;|\Delta x|=|y| \cdot \operatorname{ctg}(\beta)
$$

where:

$\Delta x, \Delta y$ - measurement errors of coordinates

$\beta$ - the tilt of the laser

$L$ - the position of the laser beam recorded by the camera sensor

$a, b, c$-geometric values (fig. 1)

$\Delta L$ - the accuracy of the camera sensor

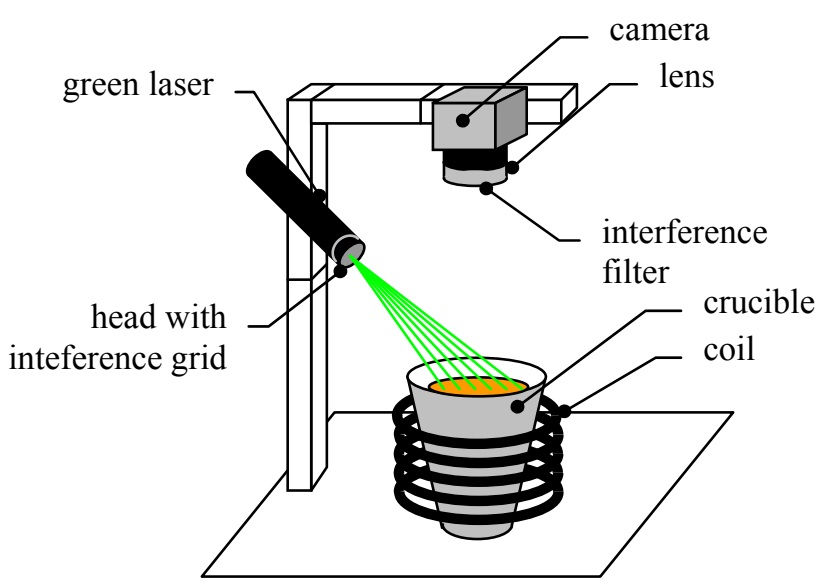

Figure 5: Experimental setup. 


\section{Conclusion}

The method of laser measurement of the surface profile may be successfully applied to record the surface of a liquid metal. The highest accuracy of results is obtained by a variant of this method in which light diffused on the metal surface is recorded. This technique avoids the problems with the influence of the surface curvature on the change in the direction of laser beam, which is why it was applied in our experimental setup.

The measurements performed with the use of the method incorporating the reflected beam allows us to calculate the curvature of the liquid metal on which the laser beam is not diffused; however, the disadvantage is the complexity and lower accuracy of the measurement.

Further studies on the issues discussed in this paper should be devoted to the expansion of the method so that the whole surface of the metal could be scanned, which would result in its application to the problems without axial symmetry.

\section{Acknowledgement}

This research work was carried out within project No. N508 034 31/1889, financially sponsored by the Polish Ministry of Science and Higher Education.

\section{References}

[1] Blacha L., Fornalczyk A., Przyłucki R., Golak S.: Kinetics of the evaporation process of the volatile component in induction stirred melts, 2nd International Conference Simulation and Modelling of Metallurgical Processes in Steelmaking STEELSIM 2007, Graz, Austria, pp. 389-395, 2007

[2] Golak S. , Przyłucki R.: Oxidation of the surface of a liquid metal in the induction furnaces., Acta Metallurgica Slovaca 13, pp. 256-259, 2007

[3] Golak S. , Przyłucki R. : The optimization of an inductor position for minimization of a liquid metal free surface, Electrotechnical Review, 11/2008, SIGMA-NOT, pp. 163-164, 2008

[4] Mass H. G., Hentschel B., Schreiber F.: An optical triangulation method for height measurements on water surfaces, Videometrics VIII (Electronic Imaging 2003), Ed. S. El Hakim, SPIE Proceedings Series Vol. 5013, pp. 103-109. 2003 